\title{
MPMI ACKNOWLEDGMENT OF REVIEWERS
}

The success of Molecular Plant-Microbe Interactions depends on the quality of manuscripts submitted by authors and on the care and competence with which they are reviewed. It is the policy of the Editorial Board to solicit reviews of manuscripts from specialists most qualified to review them. In addition to members of the Editorial Board, the individuals listed below provided constructive critical reviews of one or more manuscripts during the past year. Their names are published here in grateful appreciation for their contributions to the journal.

D. G. Adams, University of Leeds, Leeds, U.K.

J. Aguirre, Instituto de Fisiologia Celular-UNAM, Mexico

J. Alfano, University of Nebraska, Lincoln, NE, U.S.A.

P. A. H. M. Bakker, Utrecht University, Utrecht, The Netherlands

G. Bakkeren, Agriculture and Agri-Food Canada, Summerland, BC, Canada

F. Banuett, California State University, Long Beach, CA, U.S.A.

M. -A. Barny, INRA, Paris, France

C. Baron, McMaster University, Hamilton, ON, Canada

F. Barras, LCB - CNRS, Marseille, France

W. D. Bauer, Ohio State University, Columbus, OH, U.S.A.

F. C. Belanger, Rutgers University, New Brunswick, NJ, U.S.A.

A. Bent, University of Wisconsin, Madison, WI, U.S.A.

D. Bergey, Montana State University, Bozeman, MT, U.S.A.

J. Beynon, Horticulture Research International, Wellesbourne, Warwickshire, U.K.

P. R. J. Birch, Scottish Crop Research Institute, Invergowie, Dundee, U.K.

D. M. Bird, North Carolina State University, Raleigh, NC, U.S.A.

T. Bisseling, Wageningen University, Wageningen, The Netherlands

V. Blok, Scottish Crop Research Institute, Invergowie, Dundee, U.K.

M. Boelker, Universitaet Marburg, Marburg, Germany

J.-P. Bohin, Universite des Sciences et Technologies de Lille, Villeneuve d'Ascq, France

K. Boot, Clusius Laboratory, Leiden University, Leiden, The Netherlands

K. A. Borkovich, University of California, Riverside, CA, U.S.A.

G. Braus, Georg-August University, Gottingen, Germany

E. Bremer, Philipps-University, Marburg, Germany

G. Brigneti, John Innes Centre, Colney Lane, Norwich, U.K.

W. Broughton, Universite de Geneve, Geneve, Switzerland

W. Bushnell, University of Minnesota, St. Paul, MN, U.S.A.

D. Capela, INRA-CNRS, Tolosan, France

R. W. Carlson, University of Georgia, Athens, GA, U.S.A.

N. Carpita, Purdue University, W. Lafayette, IN, U.S.A.

A. Charkowski, University of Wisconsin, Madison, WI, U.S.A.

A. K. Chatterjee, University of Missouri, Colombia, MO, U.S.A.

P. Christie, University of Texas Health Science Center, Houston, TX, U.S.A.

A. C. L. Churchill, Cornell University, Ithaca, NY, U.S.A.

V. Citovsky, State University of New York, Stony Brook, NY, U.S.A.

D. B. Collinge, Royal Veterinary and Agricultural University, Frederiksberg C, Denmark
N. Collins, John Innes Centre, Colney Lane, Norwich, Norfolk, U.K.

G. Condemine, Domaine Universitaire de la Doua, Villeurbanne, France

D. Coplin, Ohio State University, Columbus, OH, U.S.A.

P. Cornelis, Vrije Universiteit Brussel, Brussels, Belgium

M. Crespi, CNRS, Gif sur Yvette, France

J. Culver, University of Maryland, College Park, MD, U.S.A.

E. L. Davis, North Carolina State University, Raleigh, NC, U.S.A.

D. A. Day, University of Western Australia, Crawley, WA, Australia

M. Delledonne, Universita' degli Studi di Verona, Verona, Italy

S. Denison, Eckard College, St. Petersburg, FL, U.S.A.

L. Deslandes, Max-Planck-Institut f. Zuechtungsforschung, Köln, Germany

Y. Dessaux, ISV-CNRS, Yvette, Cedex, France

M. B. Dickman, University of Nebraska, Lincoln, NE, U.S.A.

R. Dietrich, Syngenta Biotechnoology Inc, Research Triangle Park, NC, U.S.A.

S. P. Dinesh-Kumar, Yale University, New Haven, CT, U.S.A.

B. Ding, Ohio State University, Columbus, OH, U.S.A.,

C. Douglas, The University of British Columbia, Vancouver, B.C., Canada

M. Dow, National University of Ireland, Cork, Ireland

A. Downie, John Innes Institute, Colney Lane, Norwich, U.K.

C. D'Souza, The University of British Columbia, Vancouver, B.C., Canada

L. D. Dunkle, Purdue University, West Lafayette, IN, U.S.A.

J. Dürner, GSF - National Research Center for Environment and Health, Oberschleissheim, Germany

P. Epple, University of North Carolina, Chapel Hill, NC, U.S.A.

T. Eulgem, University of North Carolina, Chapel Hill, NC, U.S.A.

V. Ferris, Purdue University, West Lafayette, IN, U.S.A.

C. H. Foyer, Rothamsted Research, Harpenden, Herts, U.K.

B. Fritig, IBMP-CNRS, Strasbourg, France

J. S. Gantt, University of Minnesota, St. Paul, MN, U.S.A.

F. Garcia-Arenal, Ciudad Universitaria, Madrid, Spain

W. Gassmann, University of Missouri-Columbia, Columbia, MO, U.S.A.

R. Gilbertson, University of California, Davis, CA, U.S.A.

M. Gottfert, Institut für Genetik, Dresden, Germany

A. Goverse, Wageningen University, Wageningen, The Netherlands

S. M. Gray, Cornell University, Ithaca, NY, U.S.A.

J. Greenberg, University of Chicago, Chicago, IL, U.S.A. 
P. Gresshoff, The University of Queensland, Brisbane, St. Lucia, Australia

I. Grgurina, Università "LaSapienza" di Roma, Roma, Italy

D. Gross, Texas A\&M University, College Station, TX, U.S.A.

R. Grumet, Michigan State University, East Lansing, MI, U.S.A.

R. Hammerschmidt, Michigan State University, East Lansing, MI, U.S.A.

A. Hardham, The Australian National University, Canberra, Australia

S. Y. He, Michigan State University, East Lansing, MI, U.S.A.

M. Heath, University of Toronto, Toronto, ON, Canada

R. Hedrich, Universitat Wurzburg, Wurzburg, Germany

J. Helder, Wageningen University, Wageningen, The Netherlands

T. Heulin, UMR 163 CNRS-CEA, Saint Paul Lez Durance, France

B. Hirel, INRA, Versailles, France

B. A. Horwitz, Technion - Israel Institute of Technology, Haifa, Israel

R. Hueckelhoven, Justus Liebig-Universitaet Giessen, Giessen, Germany

S. Hutcheson, University of Maryland, College Park, MD, U.S.A.

M. Jahn, Cornell University, Ithaca, NY, U.S.A.

E. James, University of Dundee, Dundee, U.K.

E. Jensen, University of Aarhus, Aarhus, Denmark

D. A. Jones, Australian National University, Canberra, Australia

M. Joosten, Wageningen University, Wageningen, The Netherlands

E. -P. Journet, UMR INRA-CNRS, Castanet-Tolosan, France

P. Kachroo, University of Kentucky, Lexington, KY, U.S.A.

J. Kaemper, Max-Planck Institut, Marburg, Germany

S. Kang, The Pennsylvania State University, University Park, PA, U.S.A.

E. Kannenberg, Universitat Tubingen, Tubingen, Germany

F. Katagiri, University of Minnesota, St. Paul, MN, U.S.A.

H. Keller, INRA, UIPMSV, Antibes, France

H. C. Kistler, University of Minnesota, St. Paul, MN, U.S.A.

S. Kjelleberg, University of New South Wales, Sydney, Australia

D. F., Klessig, Boyce Thompson Institute for Plant Research, Ithaca, NY, U.S.A.

J. Klose, The University of British Columbia, Vancouver,

B.C., Canada

P. Knox, University of Leeds, Leeds, U.K.

A. Koch, Institut d'Ecologie, Université de Lausanne, Lausanne, Switzerland

R. Koebnik, Martin-Luther-University Halle-Wittenberg, Halle (Saale), Germany

K.-H. Kogel, Inst. für Phytopathologie und Angewandte Zoologie, Giessen, Germanu

A. Kohler, INRA-Nancy, Champenoux, France

L. M. Kohn, University of Toronto, Mississauga, ON, Canada

R. Kolter, Harvard Medical School, Boston, MA, U.S.A.

J. Kopka, Max Planck Institute of Molecular Plant Physiology, Golm, Germany

F. Krajinski, University Hanover, Hanover, Germany

H. B. Krishnan, University of Missouri, Columbia, MO, U.S.A.

H. Kuester, Universitat Bielefeld, Bielefeld, Germany

J. Labavitch, University of California, Davis, CA, U.S.A.
E. Lam, Rutgers State University of New Jersey, New Brunswick, NJ, U.S.A.

E. Laubert, INRA-CNRS, Castanet Tolosan, France

D. Le Rudulier, CNRS, Nice, France

J. Leach, Kansas State University, Manhattan, KS, U.S.A.

J. R. Leadbetter, California Institute of Technology, Pasedena, CA, U.S.A.

S. Leisner, The University of Toledo, Toledo, OH, U.S.A.

A. Leuchtmann, Geobotanisches Institut, ETH-Z, Zürich, Switzerland

S. B. Levery, University of New Hampshire, Durham, NH, U.S.A.

X, Li, University of British Columbia, Vancouver, Canada

J. Liu, Boyce Thompson Institute for Plant Research, Ithaca, NY, U.S.A.

G. J. Loake, University of Edinburgh, Edinburgh, Scotland

J. W. Mansfield, Wye College, Wye, Ashford, Kent, U.K.

M. A. Marahiel, Philipps University of Marburg, Marburg, Germany

S. M. Marek, Oklahoma State University, Stillwater, OK, U.S.A.

F. Martin, Inst Natl Recherche Agronomique, Champenoux, France

B. Mauch-Mani, Université de Neuchatel, Neuchatel, Switzerland

A. Maule, John Innes Institute, Colney Lane, Norwich, U.K.

T. McNellis, Pennsylvania State University, University Park, PA, U.S.A.

A. H. Meijer, Leiden University, Leiden, The Netherlands

T. Mengiste, Purdue University, West Lafayette, IN, U.S.A.

J.-P. Métraux, University of Fribourg, Fribourg, Switzerland

B. C. Meyers, University of Delaware, Newark, DE, U.S.A.

R. W. Michelmore, University of California, Davis, CA, U.S.A.

J. Michiels, Centre of Microbial and Plant Genetics, Heverlee, Belgium

A. Molina, E. T. S. Ingenieros Agronomos, Madrid, Spain

I. M. Møller, RIS $\varnothing$ National Laboratory, Roskilde, Denmark

D. C. Monte, SAIN Parque Estação Biológica, Brasília, Brazil

J. Morrissey, University College Cork, Ireland

M. B. Mudgett, Stanford University, Stanford, CA, U.S.A.

J. F. Murphy, Auburn University, Auburn University, AL, U.S.A.

C. Müssig, Max Planck Institute of Molecular Plant Physiology, Golm, Germany

W. Nasser, UMR CNRS-INSA-UCB, Villeurbanne, France

S. J. Neill, University of the West of England (UWE), Bristol, Coldharbour Lane, Bristol, U.K.

R. Nelson, Samuel Roberts Noble Foundation, Ardmore, OK, U.S.A.

M. Nicole, UMR DGPC, Montpellier, France

D. L. Nuss, University of Maryland, College Park, MD, U.S.A.

F. O'Gara, National University of Ireland, Cork (UCC), Cork, Ireland

Y. Ohashi, National Institute of Agrobiological Sciences, Kannondai, Tsukuba, Ibaraki, Japan

G. Oldroyd, John Innes Centre, Norwich Research Park, Colney, Norwich, U.K.

R. Oliver, Murdoch University, Perth, Western Australia

A. Osbourn, John Innes Centre, Colney Lane, Norwich, U.K.

E. Ostergaard, University of Aarhus, Aarhus, Denmark

F. Panabieres, INRA, Antibes, France

J. Parker, Max Planck Institute for Plant Breeding Research, Cologne, Germany 
M. S. C. Pedras, University of Saskatchewan, Saskatoon, Canada

M. A. Peñalva, Centro de Investigaciones Biológicas CSIC, Madrid, Spain

J. Perez-Martin, Centro de Investigaciones Biológicas CSIC, Madrid, Spain

M. Perlin, University of Louisville, Louisville, KY, U.S.A.

X. Perret, University of Geneva, Geneva, Switzerland

C. Pieterse, Utrecht University, Utrecht, The Netherlands

M. Plamann, University of Missouri-Kansas City, Kansas City, MO, U.S.A.

G. K. Podila, University of Alabama, Huntsville, AL, U.S.A.

P. Poole, University of Reading, Whiteknights, Reading, U.K.

A. Puehler, University Bielefeld, Bielefeld, Germany

F. Qu, Universityof Nebraska, Lincoln, NE, U.S.A.

P. Ratet, CNRS, Yvette, France

N. Requena, Botanisches Institut, Tübingen, Germany

P. Reymond, Université de Lausanne, Lausanne, Switzerland

D. Rigling, Swiss Federal Research Institute WSL,

Birmensdorf, Switzerland

D. Roby, UMR CNRS/INRA, Castanet, Tolosan, France

D. Rochon, Agriculture and Agri-Food Canada,

Summerland, BC, Canada

P. Ronald, University of California, Davis, CA, U.S.A.

C. W. Ronson, University of Otago, Dunedin, New Zealand

M. Roosinck, The Samuel Roberts Noble Foundation, Ardmore, OK, U.S.A.

J. K. C. Rose, Cornell University, Ithaca, NY, U.S.A.

G. Saalbach, RIS $\varnothing$ National Laboratory, Roskilde, Denmark

C. Saillard, Institut National de la Recherche Agonomique and Universite Victor Segalen, Villenave d'Ornon, France

B. San Segundo, Instituto de Biologia molecular de Barcelona, CID-CSIC, Barcelona, Spain

J. Sanjuán, Estacion Experimental del Zaidin, CSIC, Granada, Spain

H. Sano, Nara Institute of Science and Technology, Takayama, Ikoma, Nara, Japan

A. Schaller, Institut für Physiologie und Biotechnologie der Pflanzen, Stuttgart, Germany

D. Scheel, Institute of Plant Biochemistry, Halle, Germany

H. R. M. Schlaman, Leiden University, Leiden, The Netherlands

K.-B. Scholthof, Texas A\&M University, College Station, TX, U.S.A.

B. Scholz-Schroeder, Washington State University, Pullman, WA, U.S.A.

M. Schultze, University of York, Heslington, York, U.K.

P. Schweizer, Institute of Plant Genetics, And Crop Plant Research (IPK), Gatersleben, Germany

A. D. Shapiro, Delaware Biotechnology Institute, Newark, DE, U.S.A.

A. Sharon, Tel Aviv University, Tel Aviv, Israel

J. E. Sherwood, Montana State University, Bozeman, MT, U.S.A.

N. Shibuya, Meiji University, Kawasaki, Kanagawa, Japan

K. Shimamoto, Nara Institute of Science and Technology, Ikoma, Nara, Japan

K. Shirasu, John Innes Centre, Colney Lane, Norwich, U.K.

P. J. Slininger, USDA Agricultural Research Service, Peoria, IL, U.S.A.

C. Sohlenkamp, Centro de Investigacion sobre Fijacion de
Nitrogeno-UNAM, Cuernavaca, Morelos, Mexico

I. Somssich, Max-Planck-Institut f. Zuechtungsforschung, Köln, Germany

R. Sonti, Center for Cellular and Molecular Biology (CCMB), Hyderabad, India

D. A. St. Clair, University of California, Davis, CA, U.S.A.

G. Stacey, University of Missouri, Columbia, MO, U.S.A.

J. Stanley, John Innes Centre, Colney Lane, Norwich, U.K.

B. J. Steffenson, University of Minnesota, St. Paul, MN, U.S.A.

J. Sullivan, University of Otago, Dunedin, New Zealand

S. Taira, Biocenter Helsinki, University of Helsinki, Finland

J. Taylor, NRC, Saskatoon, Saskatchewan, Canada

G. A. Thompson, University of Arkansas at Little Rock, Little Rock, Arkansas, U.S.A.

J. Ton, Institut de Botanique, Université de Neuchâtel, Neuchâtel, Switzerland

M. A. Torres, University of North Carolina, Chapel Hill, NC, U.S.A.

S. Travella, University of Zürich, Zürich, Switzerland

P. Tudzynski, Westfalische Wilhelms University, Muenster, Germany

G. Turgeon, Cornell University, Ithaca, NY, U.S.A.

J. G. Turner, University of East Anglia, Norwich, U.K.

B. Tyler, Virginia Polytechnic and State University, Blacksburg, VA, U.S.A.

R. G. Upchurch, North Carolina State University, Raleigh, NC, U.S.A.

M. E. Urgel, Estacion Experimental del Zaidin. CSIC., Granada 18008, Spain

L. J. Vaillancourt, University of Kentucky, Lexington, KY, U.S.A.

J. Valkonen, Swedish University of Agricultural Sciences (SLU), Uppsala, Sweden

G. Van den Ackerveken, Utrecht University, Utrecht, The Netherlands

E. van der Vossen, Plant Research International, Wageningen, The Netherlands

F. Van Gijsegem, INA-PG, Paris, France

J. Vanderleyden, Katholieke Univ Leuven, Heverlee, Belgium

S. vanWees, The Scripps Research Institute, La Jolla, CA, U.S.A.

J. Verchot-Lubicz, Oklahoma State University, Stillwater, OK, U.S.A.

P. Vignais, CEA/Grenoble, DRDC/BBSI, Grenoble, France

T. Visser, Wageningen University, Wageningen, The Netherlands

S. von Bodman, University of Connecticut, Storrs, CT, U.S.A.

G. Walker, MIT, Cambridge, MA, U.S.A.

G. -L. Wang, The Ohio State University, Columbus, OH, U.S.A.

M. C. Whalen, San Francisco State University, San Francisco, CA, U.S.A.

F. White, Kansas State University, Manhattan, KS, U.S.A.

S. Whitham, Iowa State University, Ames, IA, U.S.A.

K. Willis, University of Wisconsin, Madison, WI, U.S.A.

W. Wintermantel, USDA-ARS, Salinas, CA, U.S.A.

R. Wise, Iowa State University, Ames, IA, U.S.A.

T. J. Wolpert, Oregon State University, Corvallis, OR, U.S.A.

J. -R. Xu, Purdue University, West Lafayette, IN, U.S.A. 